DOE/ER/40561-C64-INT92-07-07

\title{
Chiral Symmetry and Nucleon Structure
}

\author{
Barry R. Holstein \\ DOE/ER/40561--64 \\ Deparmen of Phusics and Astronomy \\ University of Massachusents, Amherst, MA01003 \\ DE92 040067 \\ and \\ Instimute for Nuclear Theory, HN.12 \\ University of Washington, Seattle, WA 98195
}

\section{PREPARED FOR THE U.S. DEPARTMENT OF ENERGY,
UNDER GRANT DE-FG06-90ER40561}

This report was prepared as an account of work sponsored by the United States Government. Neither the United States nor any agency thereof, nor any of their employees, makes any warranty, express or implied, or assumes any legal liability or responsibility for the accuracy, completeness, or usefulness of any information, apparatus, product, or process disclosed, or represents that its use would not infringe privately owned rights. Reference herein to any specific commercial product, process, or service by trade name, mark, manufacturer, or otherwise, does not necessarily constitute or imply its endorsement, recommendation, or favoring by the United States Government or any agency thereof. The views and opinions of authors expressed herein do not necessarily state or teflect those of the United States Government or any agency thereof. 
UMHEP. 372

\title{
CHIRAL SYMMETRY AND NUCLEON STRUCTURE ${ }^{+}$
}

\author{
BARRY R. HOLSTEIN \\ Department of Physics and Astronomy, University of Massachusetts, Amherst. MA 01003 \\ and \\ Instirute for Nuclear Theory, University of Washington, Seattle, WA 98195
}

\begin{abstract}
Recently it has been realized that signiticant tests of the validity of $Q C D$ are available in low encrgy experiments ( $E<500 \mathrm{MeV}$ ) by exploiling the property of (broken) chiral symmetry. This technique has been highly developed in the Goldstone boson sector by the work of Gasser and Leutwyler. Application 10 the nucleon system is much more difficult and is now being carefully developed.
\end{abstract}

\section{Introduction}

The past three decades have witnessed a remarkable evolution in our methods for treating hadronic interactions and structure. Indeed during the 1960's the use of symmetry and dispersive techniques was preeminent because of the lack of a believable dynarnical model. Toward the end of inat decade, with the discovery of scaling at SLAC. this situation changed dramatically. Quarks were no longer considered to be "imaginary" objects. Rather they 'were taken as "real" and this led to the publication of numeruus quark model calculations. However, toward the end of the past decade and certainly in the present one, it has become clear that in order to understand the very precise experiments which are now porsible, e.g., the EMC "proton spin content"! and the Illinois and Oak Ridge nucleon polarizability measurements, the simple valence quark calculations of the past are no longer adequate. Thus, theorists have begun once again to exploit techniques which are generally valid, independent of specific models of hadronic structure. One example is the use of heavy quark methods, as pioneered by Isgur and Wise. ${ }^{3} \mathrm{~A}$ second, developed by Gasser and Leutwyler, is the use of (broken) chiral symmery in order to test the validity of QCD. The formalism by which this invariance can be exploised is based upon an expansion in energy, momentum-it is a low energy procedure-and is well developed in the Goldstone sector. A corresponding formalism can also be developed for baryons. 5 This program is more difficult, but results of such calculations are beginning to appear and implications for nucleon structure and interactions are the subject of this talk. In

'Rescarch suppored in part by the National Scicnce Fcundation and by the Deparunent of Energy.

Invited presentation at Baryons 'Q2, :iew Haven, June 1992 
the next section then we review the idea of chiral symmetry and its implementation via effecuve Lagtangian methods. In the following section III we discuss specific implications tor the nucleon. Our results are summarized in a concluding section IV.

\section{Chiral Symmetry: Bosons}

The holy grail for knights of the paricle/nuclear physics round table for the past two decrades has been 10 verify the correctness of the QCD Lagrangian

with

$$
L_{Q C D}=-\frac{1}{2} \operatorname{Tr} G_{\mu \nu} G^{\mu \nu}+\bar{q}(i D-m) q
$$

$$
\begin{aligned}
& i D_{\mu}=\mathrm{i} \partial_{\mu}-g\left[A_{\mu}, \quad\right] \\
& G_{\mu v}=\partial_{\mu} A_{v}-\partial_{\nu} A_{\mu}-g\left[A_{\mu}, A_{v}\right]
\end{aligned}
$$

The search for the elusive grail has proven to be difficult primarily for two reasons-the inherent nonlinearity of the quarry, Eq. (1), and the feature that it is written in terms of quark, gluon degrees of freedom rather than in terms of the hadrons with which experiments are performed. Nevertheless, the quest continues apace and there exist at least two parallel programs which attempt to make rigorous contact between $L_{Q C D}$ and experiment. One uses reactions at the highest energies in order to exploit the asymptotic freedom of $Q C D$, which asserts that

$$
g\left(q^{2}\right) \underset{q^{2} \rightarrow \infty}{\longrightarrow} 0
$$

so that perturbative methods can be employed. 6 The second which is the subject of the present talk, involves experiments at the lowest end of the energy scale in order to take advantage of the chiral symmetry of $L_{\mathrm{QCD}}$.

We can understand this feature by rewriting the light quark ( $u, d, s)$ piece of Eq. (1) as

where

$$
\begin{aligned}
L_{Q C D} & =\ldots+\bar{q}_{L} i D q_{L}+\bar{q}_{R} i D q_{R} \\
& -\bar{q}_{L} m q_{R}-\bar{q}_{R} m q_{L}
\end{aligned}
$$

$$
q_{L, R}=\frac{1}{2}\left(1 \pm \gamma_{5}\right) q
$$

denote quarks of definite chirality. ${ }^{7}$ it is easy to see then that in the limit that the quark mass is neglected $(m=0)$ the Lagrangian is invariant under independent global rotations of left-and right-handed quark identities, i.e.

under

$$
L_{\mathrm{QCD}} \rightarrow L_{\mathrm{QCD}}
$$




$$
\begin{aligned}
& q_{L} \rightarrow \exp \left(i \sum \alpha_{j} \lambda_{j}\right) q_{L} \\
& q_{R} \rightarrow \exp \left(i \sum_{j} \beta_{j} \lambda_{j}\right) q_{R}
\end{aligned}
$$

which is chiral $S U(3)_{L} \otimes S U(3)_{R}$. Of course, this symmetry is spontaneously broken in the axial sector leading, according to Goldstone's theorem, ${ }^{8}$ to the existence of eight light pseudoscalar bosons- $-\pi, K, \eta$-which would be strictly massless in the $m \rightarrow 0$ limit.

The earliest use of this invariance took place in the 1960's when current algebra/PCAC enabled one to relate processes which differed by one in the number of pions (kaons) $)^{9}$

$$
\left\langle\pi_{i,}^{\mathrm{a}} \mathrm{B}|O| \mathrm{A}>\underset{\mathrm{Q} \rightarrow 0}{\rightarrow} \frac{-\mathrm{i}}{\mathrm{F}_{\pi}}<\mathrm{B}|| \mathrm{Q}_{5}^{\mathrm{a}}, O|| \mathrm{A}\right\rangle
$$

However, it was soon realized that this same information was encoded in effective chiral Lagrangians to be used at tree level. The simplest such form, describing inseractions in the Goldstone sector. is given by 10

$$
L_{\text {eff }}^{(2)}=\frac{F^{2}}{4} \operatorname{Tr} D_{\mu} U D_{H} U^{\dagger}+\frac{F_{\pi}^{2}}{4} \operatorname{Tr} 2 B_{0} m\left(U+U^{\dagger}\right) .
$$

Here

$$
U=\exp \left(\frac{i}{F_{k}} \sum_{j} \lambda_{j} \phi_{j}\right)
$$

is a highiy nonlinear function of the Goldstone fields $\phi_{j}, F_{\pi}=92.4 \mathrm{MeV}$ is the pion decay constant, ${ }^{11} \mathrm{~m}=\left(\mathrm{m}_{\mathrm{u}}, \mathrm{m}_{\mathrm{d}}, \mathrm{m}_{\mathrm{S}}\right)_{\text {diag }}$ is the quark mass matrix, and

$$
2 \mathrm{~B}_{0}=\frac{2 m_{\pi}^{2}}{m_{\mathrm{u}}+\mathrm{m}_{\mathrm{d}}}=\frac{2 \mathrm{~m}_{\mathrm{K}}^{2}}{\mathrm{~m}_{\mathrm{u}}+\mathrm{m}_{\mathrm{s}}}
$$

is a pheromenological constant. Expansion of Eq. 5 to $O\left(\phi^{2}\right)$ yields simply the free pseudoscalar Lagrangian

$$
L_{\mathrm{cff}}^{(2)}\left(\phi^{2}\right)=\sum_{j=1}^{8}\left(\frac{1}{2}\left(\partial_{\mu} \phi_{j}\right)^{2}-\frac{1}{2} \mathrm{~m}_{j}^{2} \phi_{j}^{2}\right)
$$

while $O\left(\phi^{4}\right)$ terms produce the familiar "current algebra predictions" for meson-meson scattering, e.g. the Weinberg $\pi-\pi$ scattering lengths ${ }^{12}$

$$
a_{0}^{0}=\frac{7 m_{\pi}}{32 \pi F_{\pi}^{2}} \quad a_{0}^{2}=-\frac{m_{\pi}}{16 \pi F_{\pi}^{2}} .
$$

However, the significance of such predictions is unclear, as Eq. (5) includes only two of an infinite number of possible terms. The way out of this abyss was pointed by Weirberg 13 who emphasized that Eq. 5 \} represents nearly the lowest onder term in an 
expansion in derivatives (energy-momentum) and in chiral symmerry breaking (pseudo scalar mass terms) with a cniral scale parameter ${ }^{\text {lit }}$

$$
\Lambda_{\chi}-4 \pi F_{\pi}-1 \mathrm{GeV}
$$

measuring the importance of higher order effects-a four derivative term such as $\operatorname{Tr} D_{\mu} \mathrm{UD}^{\mu} \mathrm{U}^{\dagger} \mathrm{D}_{v} \cup \mathrm{D}^{\vee} \mathrm{U}^{\dagger}$ is suppressed compared to its swo derivative counterpar in Eu. (5) by terms of $O\left(E^{2} / \Lambda_{\chi}^{2}\right)$. Thus this is a low energy method. Extension to higher energy san be performed by inclusion of loop contributions from $L^{(2)}$ in order to enforce unitarity together with a complete set of chiral counterterms of $O\left(E^{4}, E^{2} m^{2}, m^{4}\right)$ in order absorb the various divergences which might arise. The form of this $O\left(E^{4}\right)$ Lagrangian has been given by Gasser and Leutwyler

$$
\begin{gathered}
L^{(4)=} L_{1}\left(\operatorname{Tr} D_{\mu} \cup D^{\mu} U^{\dagger}\right)^{2}+L_{2}\left(\operatorname{Tr} D_{\mu} U D_{\nu} U^{\dagger}\right)^{2} \\
+\ldots+L_{10} \operatorname{Tr} F_{\mu \nu}^{L} \cup \text { FHVR } U^{\dagger}
\end{gathered}
$$

in terms of ten phenomenological constants $L_{1}, \ldots L_{10}$ which have been determined empirically as shown below.

$$
\begin{array}{ccccc}
\mathrm{L}_{1}^{\mathrm{r}} & \mathrm{L}_{2}^{\mathrm{r}} & \mathrm{L}_{3}^{\mathrm{r}} & \mathrm{L}_{9}^{\mathrm{r}} & \mathrm{L}_{10}^{\mathrm{r}} \\
0.71 \pm 0.28 & 2.01 \pm 0.37 & 2.7 \pm 0.3 & 7.7 \pm 0.2 & -5.2 \pm 0.3
\end{array}
$$

Table I: Empirical Values of Chiral Expansion Parameters $\left(\times 10^{-3}\right.$ with $\mu=$ on $\left.\eta\right)$

This procedure for understanding the interactions of Goldstone bosons has been highly developed by Gasser, Leutwyler and others and leads to a highly successful (and succinct) description of such processes. It is even predictive, leading, for example to highly nontrivial tests for the validity of chiral symmetry and thereby of QCD itself. ${ }^{15}$ Examples include

i) a prediction for the size of the charged pion electric and magnetic polarizabilities in terms of the measured axial structure constant $h_{A}$ in radiative pion decay $\pi \rightarrow$ evplts

ii) a prediction for the axial structure function $r_{A}$ in the Dalitz pion decay $\pi \rightarrow$ evee in terms of the rneasured pion charge radius,

etc.

It is then of great interest to see whether corresponding understanding can be gleaned within the baryon sector, and this will be the subject which occupies us for the remainder of this presentation. 


\section{Chiral Symmetry: Baryons}

Generalization of the chiral perturbative formalism to the meson-nucleon system begins straightforwardly- the lowest order (zero- and one-derivative) Lagrangian is found to be 5

$$
L^{(1)}=\bar{\psi}(x)\left(i D-\dot{M}+i \dot{g}_{A} \gamma^{\mu} \gamma_{s} \Delta_{\mu}\right) \psi(x)
$$

where here $\stackrel{\circ}{M} . \stackrel{0}{g} A$ represent the nucleon mass, axial coupling constant in the chirallimit. Also defining $U=\xi^{2}$ we have

$$
\begin{gathered}
\Delta_{\mu}=\frac{1}{2} \xi^{\dagger} \nabla_{\mu} \cup \xi^{\dagger} \\
D_{\mu}=\partial_{\mu}+\Gamma_{\mu} \quad \text { with } \quad \Gamma_{\mu}=\frac{1}{2}\left[\xi^{\dagger}, \partial_{\mu} \xi\right]
\end{gathered}
$$

Even at this level, there is predictive power. Indeed, using the nucleon equation of motion, we find the $\pi N N$ coupling $g$ to be given by

$$
\dot{g}=\frac{\dot{M} \dot{g}_{A}}{\dot{F}}
$$

which is the Goldberger-Treinan relation. ${ }^{17}$ For future use, it is uset ui to note that Eq. (14) is easily derived using the PCAC condition in the chiral limit

$$
\partial_{\mu} A_{a}^{\mu}=0 \text {. }
$$

Writing

$$
\left.<p\left|A_{1 !}^{+}\right| n\right\rangle=\bar{u}\left(p_{2}\right)\left(\dot{g}_{A} \gamma_{\mu} \gamma_{5}+\dot{g}_{p} \frac{q_{\mu}}{2 \dot{M}} \gamma_{5}\right) u\left(p_{1}\right)
$$

and assuming pion pole dominance for the induced pseudoscalar

$$
s_{p}=\frac{\sqrt{2} 2 \dot{M} \dot{F}}{q^{2}} \times \sqrt{2} \dot{g}
$$

we find, taking the divergence

$$
\begin{aligned}
& <p\left|\gamma^{\mu} A_{\mu}^{+}\right| n>=i \bar{u}\left(p_{2}\right) \gamma_{S} u\left(p_{1}\right)\left[2 \dot{M} \dot{g}_{A}-2 \dot{F} \dot{g}\right]=0 \\
& \text { i.e., } \quad \dot{M} \dot{g}_{A}=\dot{F} \dot{g} .
\end{aligned}
$$

Interestingly, the form of this relation remains unchanged even when chiral symmetry is broken so that

$$
\partial \mu A_{\mu}^{2}=F_{\pi} m_{\pi}^{2} \phi_{\pi}^{a}
$$

Eq. (17) then is replaced by

$$
g_{p}=\frac{\sqrt{2} 2 M F_{\pi}}{q^{2}-m_{\pi}^{2}} \times \sqrt{2} g
$$


while Eq. (18) becomes

$$
\begin{aligned}
& \begin{aligned}
<p\left|\partial^{\mu} A_{\mu}^{+}\right| n> & =i \bar{u}\left(p_{2}\right) \gamma_{5} u\left(p_{1}\right)\left[2 M g_{A}-2 F_{\pi} g \frac{q^{2}}{q^{2}-m_{\pi}^{2}}\right] \\
& =-i \bar{u}\left(p_{2}\right) \gamma_{5} u\left(p_{1}\right) F_{\pi} m_{\pi}^{2} g \frac{1}{q^{2}-m_{\pi}^{2}}
\end{aligned} \\
& \text { i.e., } \quad M g_{A}\left(q^{2}\right)=F_{\pi} g\left(q^{2}\right)
\end{aligned}
$$

as before. However, the way in which this result obtains in the effective Lagrangian approach is more subtle and involves a careful study of one loop corrections to these lowest order quantities as well as inclusion of a set of counterterms ${ }^{5}$

$$
L^{(1)} \rightarrow L(1)+\Delta
$$

with

$$
\Delta \mathrm{L}=\Delta \dot{\mathrm{m}} \frac{\dot{\mathrm{m}}^{2}}{\dot{\mathrm{F}}^{2}} \bar{\psi} \psi-\Delta \dot{\mathrm{g}}_{\mathrm{A}} \frac{\dot{\mathrm{m}}^{2}}{2 \dot{\mathrm{F}}^{2}} \psi \gamma_{s} \gamma_{\mu} \mathrm{u}^{\mu} \psi+\ldots .
$$

When this program is carried out one finds that

$$
M g_{A}(0)=F_{\pi} g(0)
$$

in agreement with Eq. (18). However, testing this relation involves a bit of effort in that experiment gives us the value $g\left(\mathrm{~m}_{\pi}^{2}\right)$ not $g(0)$. Thus corrections are expected and chiral perturbation theory does not predict the size of such corrections. ${ }^{18}$ Writing

$$
1-\frac{M g_{A}(0)}{F_{\pi} g\left(m_{\pi}^{2}\right)}=\Delta_{\pi}
$$

we have

$$
\Delta_{\pi}=1-\frac{g(0)}{g\left(m_{\pi}^{2}\right)}
$$

One can estimate $\Lambda_{\pi}$ using a Feynman diagram as in Figure 1

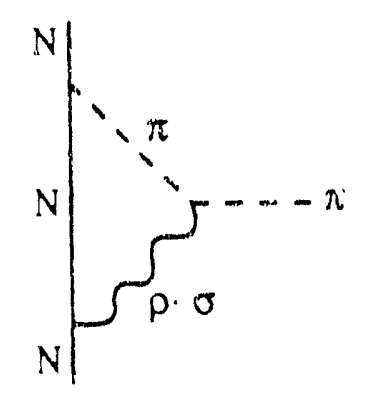

Figure ! 
which yields 18

$$
\Delta_{\pi}-\frac{m_{\pi}^{2}}{2 m_{\rho}^{2}} \sim 0.017
$$

In comparison with experiment only the nucleon mass has been stable over the past few years. The size of the axial coupling has increased over the years and the present value is 19

$$
g_{A}=1.262 \pm 0.004
$$

The size of $F_{\pi}$ has recently decreased by about a percent due to a reevaluation of the radiative correction and the present value is $\$ 1$

$$
F_{\pi}=92.4 \pm 0.3
$$

The most uncertainty lies with the size of the pion-nucleon coupling constant, where there exists a systematic difference berween older and newer values as shown in the numbers below.

$\begin{array}{ccc} & g^{2} / 4 \pi & \Delta_{\pi} \\ \pi^{ \pm} & 13.31(27)^{20} & 0.002 \\ & 14.28(18)^{21} & 0.043 \\ \pi^{0} & 13.55(13)^{22} & \\ & 14.52(40)^{23} & 0.017 \\ & & \end{array}$

Clearly the "new" values agree with the estimate given in Eq. (27) while the "old" numbers appear too large, although it should be einphasized that Eq. (27) is not a prediction of chiral symmerry.

A second indication of possible problems here can be found by extending this discussion to $\mathrm{SU}(3)$, and studying the $\Lambda \mathrm{N}$ and $\Sigma N$ couplings, which are shown below. 24

$$
\mathrm{g}\left(\mathrm{m}_{\mathrm{K}}^{2}\right) \quad \mathrm{g}_{\mathrm{A}}
$$

$$
\begin{array}{lll}
\Lambda & 13.1 \pm 0.9 & 0.70 \pm 0.03 \\
\Sigma & -6.8 \pm 1.2 & -0.34 \pm 0.05
\end{array}
$$

These can also be used as a test of the Goldberger-Treiman relation, yielding

$$
\Delta_{K}=1-\frac{\left(M_{N}+M_{\Sigma \Lambda}\right) g_{A}(0)}{\sqrt{2} F_{K} g\left(m_{K}^{2}\right)}= \begin{cases}0.32 \pm 0.05 & \Lambda \\ 0.34 \pm 0.15 & \Sigma\end{cases}
$$

Clearly the discrepancy is larger, but this is expected. Indeed, using a theorem due to Dashen and Weinstein, the parameter $\Delta$ can be parameterized in terms of the relevant quark mass tirnes an SU(3) octet operator, i.e., 25 


$$
\begin{aligned}
& \left(m_{N}+m_{p}\right) g_{A}^{n p}=2 F_{\pi} g_{\pi}+\left(m_{u}+m_{d}\right)(f+d)+O\left(m^{2}\right) \\
& \left(m_{A}+m_{p}\right) g_{A}^{\Lambda p}=-\sqrt{2} F_{K} g_{\Lambda}-\left(m_{u}+m_{s}\right) \sqrt{\frac{3}{2}}\left(f+\frac{1}{3} d\right)+O\left(m^{2}\right) \\
& \left(m_{\Sigma}+m_{n}\right) g_{A}^{\Sigma n}=\sqrt{2} F_{K} g_{\Sigma}-\left(m_{u}+m_{s}\right)(f-d)+O\left(m^{2}\right) .
\end{aligned}
$$

These relations obey the sum rule

$$
\Delta_{\pi}=\frac{\sqrt{3}}{2} \frac{F_{K}}{F_{\pi}} \frac{m_{u}+m_{d}}{m_{u}+m_{s}}\left(\frac{g_{\Lambda}}{g_{\pi}} \Delta_{K}^{\Lambda}-\frac{1}{\sqrt{6}} \frac{g_{\Sigma}}{g_{\pi}} \Delta_{K}^{\Sigma}\right) .
$$

Using $\left(m_{u}+m_{d}\right) /\left(m_{u}+m_{s}\right)=m_{\pi}^{2} / m_{K}^{2}$ we find

$$
\Delta_{\pi}^{\text {theo }}=0.030
$$

which lies between the "old" and "new" values for $g_{\pi N N}$. Clearly it is high time to find consistent values for $g_{\pi N N}$ and to put this issue to rest.

Another longstanding issue in this regard has to do with the so-called $\pi \mathrm{N}$ sigma term defined as

$$
\begin{aligned}
& \sigma \equiv \frac{1}{2 m_{N}}<p|\hat{m}(\bar{u} u+d d)| p> \\
& \hat{m}=\frac{1}{2}\left(m_{u}+m_{d}\right) .
\end{aligned}
$$

According to a well-known current algebra theorem, $\sigma$ is identical (in the chiral limit) to the iso-spin even $\pi \mathrm{N}$ scattering amplitude at the Cheng-Dashen point ${ }^{26}$

$$
\Sigma=F_{\pi}^{2} \bar{D}+\left(2 \mu^{2}\right) .
$$

The interest here lies in the fact that the value $\Sigma=(64 \pm 8) \mathrm{MeV}$ obtained from the analysis of $\pi \mathrm{N}$ scattering data 27 disagrees rather substantially with the value $\sigma \equiv 25 \mathrm{MeV}$ obtained from analysis of the baryon mass spectrum. More precisely from the mass spectrum one determines 28

$$
\begin{aligned}
& m_{\Xi}+m_{\Sigma}-2 m_{N}=\left(m_{s}-\hat{m}\right)<N|\bar{u} u+d d-2 \bar{s} s| N> \\
& \text { i.e. } \quad 634 \mathrm{MeV}=\sigma\left(\frac{m_{s}}{\hat{m}}-1\right)(1-y)
\end{aligned}
$$

where

$$
y=\frac{\langle N|2 \bar{s} s| N\rangle}{\langle N|\bar{u} u+d d| N\rangle}
$$

provides a measure of the nucleon sirangeness content. Taking $m_{g} / \hat{m} \cong 25$ from the pseudoscalar mass spectrum and assuming $y=0$ from a constituent quark picture we find 


$$
\sigma=\frac{634 \mathrm{MeV}}{24} \equiv 25 \mathrm{MeV}
$$

as claimed above. In order to resolve this apparent problem a number of solutions have been proposed. One solution is that the linear mass formula in the baryon sector is invalid. However, this seems unlikely in view of the success of the Gell-Mann-Okubo mass formula 29

$$
2259 \mathrm{MeV}=m_{\Xi}+m_{N}=\frac{1}{2}\left(m_{\Sigma}+3 m_{\Lambda}\right)=2272 \mathrm{MeV} \text {. }
$$

Another possibility is that $y \equiv 0.6$. However, this seems a rather large value and would require that $\sim 400 \mathrm{MeV}$ of the nucleon's $940 \mathrm{MeV}$ mass arises from its strange quark component!

Recent work, however, by Gasser, Leutwyltr and Sainio has led to a resolution of this apparent paradox. ${ }^{30}$ Brown, Pardee and Peccei have shown that away from the chiral limit one has ${ }^{31}$

$$
\Sigma=\sigma\left(2 \mu^{2}\right)+O\left(\hat{\mathrm{m}}^{2}\right)
$$

The value of $\sigma\left(2 \mu^{2}\right)$ can be estimated by means of a dispersion relation and is found to be quite large

$$
\sigma\left(2 \mu^{2}\right)-\sigma(0)=15 \mathrm{MeV}
$$

due to the importance of the $I=0 \pi \pi$ channel. ${ }^{32}$ In addition higher order chiral effects have been estimated to yield

We find then

$$
\delta \sigma(0) \cong 10 \mathrm{MeV}
$$

$$
\begin{aligned}
\sigma(0) & =\Sigma-\delta \sigma(0)-\sigma \\
& \cong 45 \mathrm{MeV}
\end{aligned}
$$

corresponding to a strangeness quark content

$$
y \equiv 0.3
$$

which contributes about $130 \mathrm{MeV}$ to the nucleon mass. This value is a reasonable one and should not be unexpected. Of course, within a constituent quark picture one might expect y $\cong 0$. However, if a kaon cloud contribution is included, as in figure 2

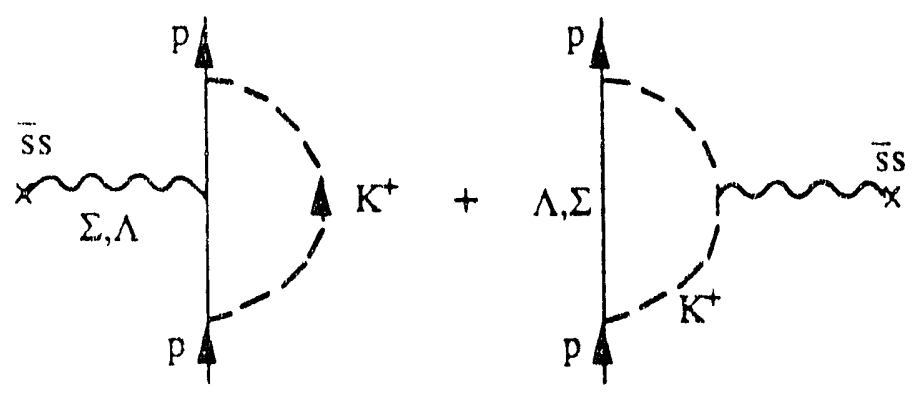

Figure, 2-Kann cloud contributions to $\langle\mathrm{N}|\bar{s} s| \mathrm{N}\rangle$. 
one would expect to find a non-zero strangeness content. ${ }^{33}$ Thus the $\sigma$ term problem may be said to be resolved. with the result that there does exist a non-zero, but not large, value for the strangeness content.

Another area of recent interest with respect to nucleon structure has to do with the polarizability, which measures the constant of proportionality between the induced electric (magnetic) moment and the applied electric (magnetizing) field ${ }^{34}$

$$
p=4 \pi \bar{\alpha}_{E} E \quad \mu=4 \pi \bar{\beta}_{M} H \text {. }
$$

Recently values of the polarizability for both neutron and proton were obtained by measurements at Qak Ridge and Illinois respectively, 35,36

$$
\begin{array}{ll}
\bar{\alpha}_{E}^{n}=(12.3 \pm 1.5 \pm 2.0) \times 10^{-4} \mathrm{fm}^{3} & \beta_{M}^{n}=(3.5 \mp 1.5 \mp 2.0) \times 10^{-4} \mathrm{fm}^{3} \\
\bar{\alpha}_{E}^{p}=(10.9 \pm 2.2 \pm 1.4) \times 10^{-4} \mathrm{fm}^{3} & \beta_{M}^{p}=(3.3 \mp 2.2 \mp 1.4) \times 10^{-4} \mathrm{fm}^{3}
\end{array}
$$

and it is of interest to see how these results can be understood.

At the level of the nonrelativistic constituent quark model, Eq. (48) cannot be understood. Indeed, making the usual nonrelativistic decomposition of the Compton polarizability $\bar{\alpha}_{E}, \vec{\beta}_{M}$ in terms of static $\left(\alpha_{E}, \beta_{M}\right)$ and recoil $\left(\Delta \alpha_{E}, \Delta \beta_{M}\right)$ components ${ }^{37}$

$$
\begin{aligned}
\Delta \alpha_{E}=\frac{\alpha}{3 m_{N}}<\sum_{i} e_{i}\left(r_{i}-R_{C M}\right)^{2}>\quad \alpha_{E}=2 \alpha & \sum_{n \neq 0} \frac{|<n| \sum_{i} e_{i}\left(r_{i}-R_{C M}\right)|0>|^{3}}{E_{n}-E_{0}} \\
\Delta \beta_{M}=-\frac{\alpha}{2 M}<\left(\sum_{i} e_{i}\left(r_{i}-R_{C M}\right)\right)^{2}>\beta_{M} & =2 \alpha \sum_{n \neq 0} \frac{|<n| \sum_{i} \frac{e_{i}}{2 m_{i}} \sigma_{i z}|0>|^{2}}{E_{n}-E_{0}} \\
& -\frac{\alpha}{6}<\sum_{i} \frac{e_{i}^{2}}{m_{i}}\left(r_{i}-R_{C M}\right)^{2}>,
\end{aligned}
$$

then in a nonrelativistic constituent quark model, even with spin-spin interactions included one finds 34

$$
\alpha_{E}^{p}=\alpha_{E}^{n} \quad \bar{\alpha}_{E}^{p}-\bar{\alpha}_{E}^{n} \cong-4.2 \times 10^{-4} \mathrm{fm}^{3}
$$

Likewise, assuming dominance by $T=\frac{3}{2}$ intermediate states, such as the $\Delta$, in the sum we find

$$
\beta_{M}^{p}=\beta_{M}^{n} \quad \bar{\beta}_{M}^{p} \equiv-2.8 \times 10^{-4} \mathrm{fm}^{3}
$$

neither of which appears to match onto the experimental findings, which imply 


$$
\bar{\alpha}_{E}^{n} s \bar{\alpha}_{E}^{p} \quad \bar{\beta}_{M}^{n}=\bar{\beta}_{M}^{p}>0
$$

As in the case of the strangeness content, a way out of this problem can be found by inclusion of a meson cloud contribution, as shown in Figure 3.38

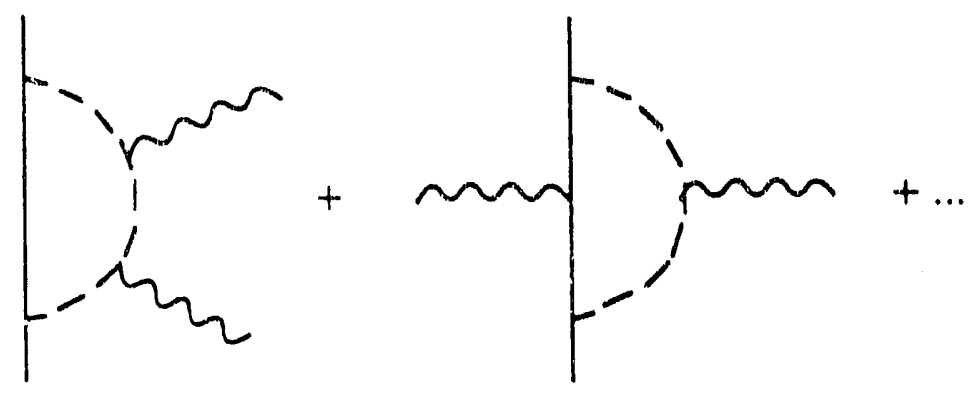

Figure 3-Meson cloud contributions to the nucleon polarizability.

Such effects can be included systematically by using the techniques of chiral perturbation theory, as outlined above. The calculation of the polarizability is a highly nontrivial task involving the evaluation of fifty-two separate Feynman diagrams! No counterterms contribute and the results are 39

$$
\begin{aligned}
& \bar{\alpha}_{E}^{p}=D\left(\frac{5 \pi}{2 \mu}+18 \ln \mu+\ldots\right)=7.9 \times 10^{-4} \mathrm{fm}^{3} \\
& \bar{\alpha}_{E}^{n}=D\left(\frac{5 \pi}{2 \mu}+6 \ln \mu+\ldots\right)=11.0 \times 10^{-4} \mathrm{fm}^{3} \\
& \bar{\beta}_{M}^{p}=D\left(\frac{\pi}{4 \mu}+18 \ln \mu+\ldots\right)=-2.3 \times 10^{-4} \mathrm{fm}^{3} \\
& \bar{\beta}_{M}^{n}=D\left(\frac{\pi}{4 \mu}+6 \ln \mu+\ldots\right)=-2.0 \times 10^{-4} \mathrm{fm}^{3}
\end{aligned}
$$

where $\mathrm{D}=\mathrm{e}^{2} \mathrm{~g}_{\mathrm{A}}^{2} / 192 \pi^{3} \mathrm{~F}_{\pi}^{2} \mathrm{~m}_{\mathrm{N}}$. We see that results are consistent with the experimental findings Eq. (48) but that the magnetic polarizability has the wrong sign! This $\vdots 3$ to be expected, however, as the $\Delta$ contribution is not included in the above, being a two loop effect. More work is needed here, but preliminary indications are encouraging.

Our final topic to which chiral methods have been applied is that of threshold pion photoproduction. ${ }^{48}$ In the case of the charged modes - $\gamma p \rightarrow \pi^{+} n, m \rightarrow \pi p$ - there is no problem. In the soft pion limit, we have ${ }^{41}$ 


$$
\left\langle\pi_{q}^{*} N\left|v_{\mu}^{e m}\right| N\right\rangle \underset{q \rightarrow 0}{\rightarrow}+\frac{i}{\sqrt{2} F \pi}\left\langle N\left|A_{\mu}^{+}\right| N\right\rangle
$$

which leads to a predicted $E_{0+}$ multipole ${ }^{42}$

$$
E_{0-\mu}=\sqrt{2} C\left\{\begin{array}{l}
1-\frac{3}{2} \mu+O\left(\mu^{2}\right)=26.3 \times 10^{-3} n_{\pi}^{-1} \\
-1+\frac{1}{2} \mu+O\left(\mu^{2}\right)=-31.3 \times 10^{-3} \mathrm{~m}_{\pi}^{-1} \quad \pi^{-} p
\end{array}\right.
$$

where $C=e g / 8 \% m_{N}$, in good agreement with the experimental results 43

$$
E_{0+}=\left\{\begin{array}{l}
27.9 \pm 0.5 \times 10^{-1} \mathrm{~m}_{\pi}^{-1} \\
-31.4 \pm 1.3 \times 10^{-3} \mathrm{~m}_{\pi}^{-1}
\end{array}\right.
$$

Although these results were obtained via at relative; large extrapolation to threshold and should be repeated, there is litule controversy in the charged sector.

On the contrary, in the case $u^{4}$ the neutual pion reaction $\gamma \rightarrow \pi$ p there has been a great deal of recent activity. Without ging into detail (early experimental claims of significant violation of the so-called low energy theorems (L.ET) have been modified, both Saclay and Mainz results yield 44,45

$$
E_{0+}^{\exp }\left(\pi^{0} \mathrm{pp}\right)=(-2.0 \pm 0.2) \times 10^{3} \mathrm{~m}_{\pi}^{-1}
$$

in excellent agreement with the LET

$$
E_{0+}^{\text {the }}(\pi \mathrm{p})=-C\left(\mu-\frac{1}{2} \mu^{2}\left(3+\kappa_{\mathrm{p}}\right)+O\left(\mu^{3}\right)\right)=-2.3 \times 10^{-3} \mathrm{~m}_{\pi}^{-1}
$$

The LET themselves are obiained by use of the Born diagrams arising from Eq. (12), as these can be shown to be consistent with both galige invariance and current algebra/PCAC constraints. ${ }^{42}$

However, recently this situation has changed in that the additional one-loop and counterterm contributions to the threshold predictions have also been calculated. Once again this is a major task, requiring the evaluation of more than sixty separate Feymman diagrams! When this is done, one finds that the charged predictions are unaffected. However, for $\gamma p \rightarrow \pi^{0} p$ it is found thar 46

$$
\begin{aligned}
& E_{D_{+}}^{\left(x_{x}\right.}\left(\mu^{0} p\right)=E_{D_{+}}^{L E T T}\left(\pi O_{p}\right)+C \frac{1}{2} \mu^{2} \frac{m_{N}^{2}}{8 k_{\pi}^{2}}+\ldots \\
& =-1.3 \times 10.3 \mathrm{~m}_{\pi}^{-1}
\end{aligned}
$$

which is now in disagreement with the experimerial results-Eq. (57). The origin of this additional contritution lies in the so-called rescattering diagrams (Figure 4) which are singular in the chiral limis. 


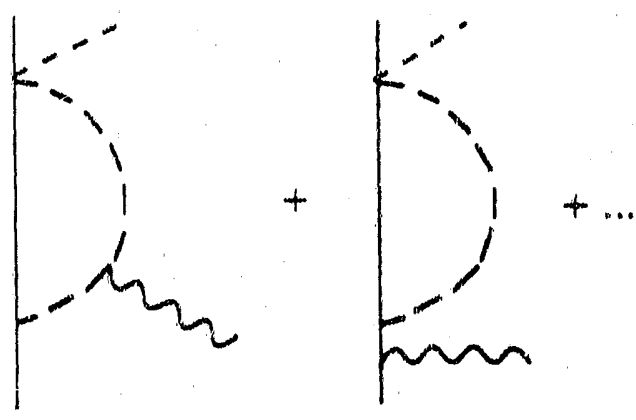

Figure 4-Rescattering contributions wo pion photoproduction.

These diagrams each contain the $\bar{N} N \pi \pi$ vertex required by chiral invariance and their importance was not realized previously because such vertices were not considered.

Clearly more work is required here. On the theoretical side, it is important to confirm the chiral calculations and to include $S U(2)$ breaking effocts, as a strong energy dependence is expected away from threshold and this must be understood in a realistic calculation. ${ }^{27}$ Experimentally, new $p \rightarrow \pi^{0} p$ measurements in the threshold region are called for in order to have a completely reliable data base. In addition it would be extremely useful to obtain a threshold $\gamma n \rightarrow \pi^{0}$ n experiment, as here 100 a supposedly reliable theoretical predition is available

$$
E_{0+}\left(\pi^{0} n\right)=-C \frac{1}{2} \mu^{2}\left(\kappa_{n} \cdot \frac{M^{2}}{8 F_{n}^{2}}\right)+\ldots=0.6 \times 10^{-3} \mathrm{~m}_{\pi}^{-1}
$$

though a substantial energy dependence is expected 48 Finally, it is important to note that LET for electroproduction predictions are also available and should provide a rich lode for future work. 49

\section{Conclusions}

We have studied here implications of (broken) chiral invariance in the arena of nucleon interactions. In the case of Goldberger-Th. whan violations, the 0 term and the nuclear polarizability experimenta' "esults are in reasonable agreement with chiral expectations, although clearing up the present disagrement with respect to the size of the pion-nucleon coupling is essential before definitive conclusions are drawn from the GT results. The situation for threshold $\pi^{0}$ photoproduction is not as clear and calls for considerable additional work, both theoretical and experinental.

\section{References}

1. I. Ashman et. al., Phys. Lett. 206 B (1988) 364; Nucl. Phys. 8328 (1989) 1.

2. J. Schmiedmyer et. al., Phys. Rev. Lett. 66 (1991) 1015:

F.J. Federspiel et. al, Phys. Ref. Lett. 67 (1991) 1511. 
3. N. Isgur and M. Wise, Phys. Lett. 232B (1989) 113: 237B (1990) 527: Phys. Rev. D41 (1990) 151.

4. J. Gasser and H. Leutwyler, (a) Ann. Phys. (NY) 158 (198.4) 142; (b) Nucl. Phys. B250 (1985) 465; (c) B250 (1985) 517.

5. J. Gasser, M.E. Sainio and A. Svarc, Nucl. Phys. B307 (1988) 779.

6. H.D. Politzer, Phys. Rev. Lett. 30 (1973) 1346 and Phys. Rept 14C (1974) 274; D.J. Gross and R. Wilczek, Phys. Rev. Lett. 30 (1973) 1343 and Phys. Rev. D8 (1973) 3633.

7. In the massless (or high erergy) limit the chirality corresponds to helicity.

8. J. Goldstone, Niovo Cimento 19 (1961) 154;

J. Goldstone, A. Saiam ant S. Weinberg, Phys. Rev. 127 (1961) 965.

9. S. Adler, Current Algebras and Applications to Purticle Physics, W.A. Benjamin, Reading, MA (1968).

10. A review of this method giving the state of the art ca-1970 can be found in S. Gasiorowicz and D.A. Geffen, Rev. Mod. Phys. 41 (1969) 531.

11. B.R. Holstein, Phys. Lett. 244B (1990) 83.

12. S. Weinberg, Phys. Rev. Lett. 17 (1966) 616.

13. S. Weinberg, Physica A96 (1979) 397.

14. A. Manohar and H. Georgi, Nucl. Phys. B 234 (1984) 189;

J.F. Donoghue, E. Golowich and B.R. Holstein, Phys. Rev. D30 (1984) 587.

15. Z.F. Donoghue aum A. Holstein, Phys. Rev. D40 (1989) 2378; D40 (1988) 3700 .

16. B.R. Holseein, Comm. Nucl. Part. Phys. 19 (1990) 221.

17. M.L. Goldberger and S.B. Treiman, Phys. Rev. 110 (1958) 1478.

18. C.A. Dominguez, Riv. del Nicovo Cemenro 8 (1985) 1.

19. P. Bopp et. al, Phys. Rev. Lett. 56 (1986) 919;

E. Klemt et. al., Z. Phys, C37 (1988) 179.

20. R. Arndt et. al., Phys. Rev. Lett. 65 (1990) 157.

21. R. Koch and E. Pieterinen, Nucl. Phys. A336 (1980) 331.

22. J.R. Bergervoet et. al., Phys. Rev. C41 (1990) 1435.

23. P. Kroll, in Plyys. Data. Vol. 22-1, ed. H. Behrens and G. Ebel (Fachinformationszentrum, Karlsruhe, 1981).

24. C.A. Dominguez, ref. 18;

N. Fuchs, H. Sazdjian and J. Stern, Phys. Leth, $238 B$ (1990) 380 and references therein.

25. R. Dashen and M. Weinstein, Phys. Rev. 188 (1969) 2330.

26. T.P. Cheng and R. Dashen, Phys. Rev. Lett. 26 (1971) 594.

27. R. Koch, Z. Phys. C15 (1962) 161.

28. J.F. Donoghue and C.R. Nappi, Phys. Lett. 168B (1986) 105.

29. M. Gell-Mann, Cal Tech Rept CTSL.20 (1961;

S. Okubo, Prog. Theo. Phys. 27 (1962) 949.

30. J. Gasser, H. Leutwyler and M.E. Sainio, Phys. Lett. 253B (1991) 252.

31. L.S. Brown, W.J. Pardee and R.D. Peccei, Phys. Rev. D4 (1971) 2801. 
32. J. Gasser, H. Leutwyler and M.E. Sainio, Phys, Lett. 253B (1991) 260.

33. B... Holstein, in Parity Violation in Electron Scattering, ed. E. Beise and R. Mckeown (World Scientific, Singapore, 1990).

34. See, e.g., B.R. Holstein. Comm. Nucl. Part. Phys., to be published.

35. J. Schmiedmyer et. al., Phys. Rev. Lett. 66 (1991) 1015.

36. F.J. Federspiel et. al., Phys. Rev. Lett. 67 (1991) 1511.

37. J.L. Friar, Ann. Phys. (NY) 95 (1975) 170.

38. R. Weiner and W. Weise, Phys. Lett. 159B (1975) 170.

39. V. Bernard, N. Kaiser and U. Meissner, Phys. Lett. 66 (1991) 1515.

40. For a recent review see D. Drechsel and L. Tiator, J. Phys. G18 (192) 449.

41. N.M. Kroll and M.A. Ruderman, Phys, Rev, 93 (1954) 233.

42. P. de Baenst, Nucl. Phys. B24 (1970) 633.

43. J.P. Burg, Ann de Phys. (Paris) 10 (1965) 363.

44. E. Mazzucato et. al., Phys. Rev. Lett. 57 (1986) 3144.

45. R. Beck et. al., Phys. Rev. Lett. 65, (1990) 1841.

46. V. Bernard, J. Gasser, N. Kaiser and U. Meissner, Phys. Rev. Lett.

47. A.M. Bernstein and B.R. Holstein, Comm. Nucl. Part. Phys. 20 (1991) 197.

48. J. Bergstrom, Phys. Rev. C44 (1991) 1768.

49. S. Sherer and J. Koch, Nucl. Phys. A534 (1991) 461. 

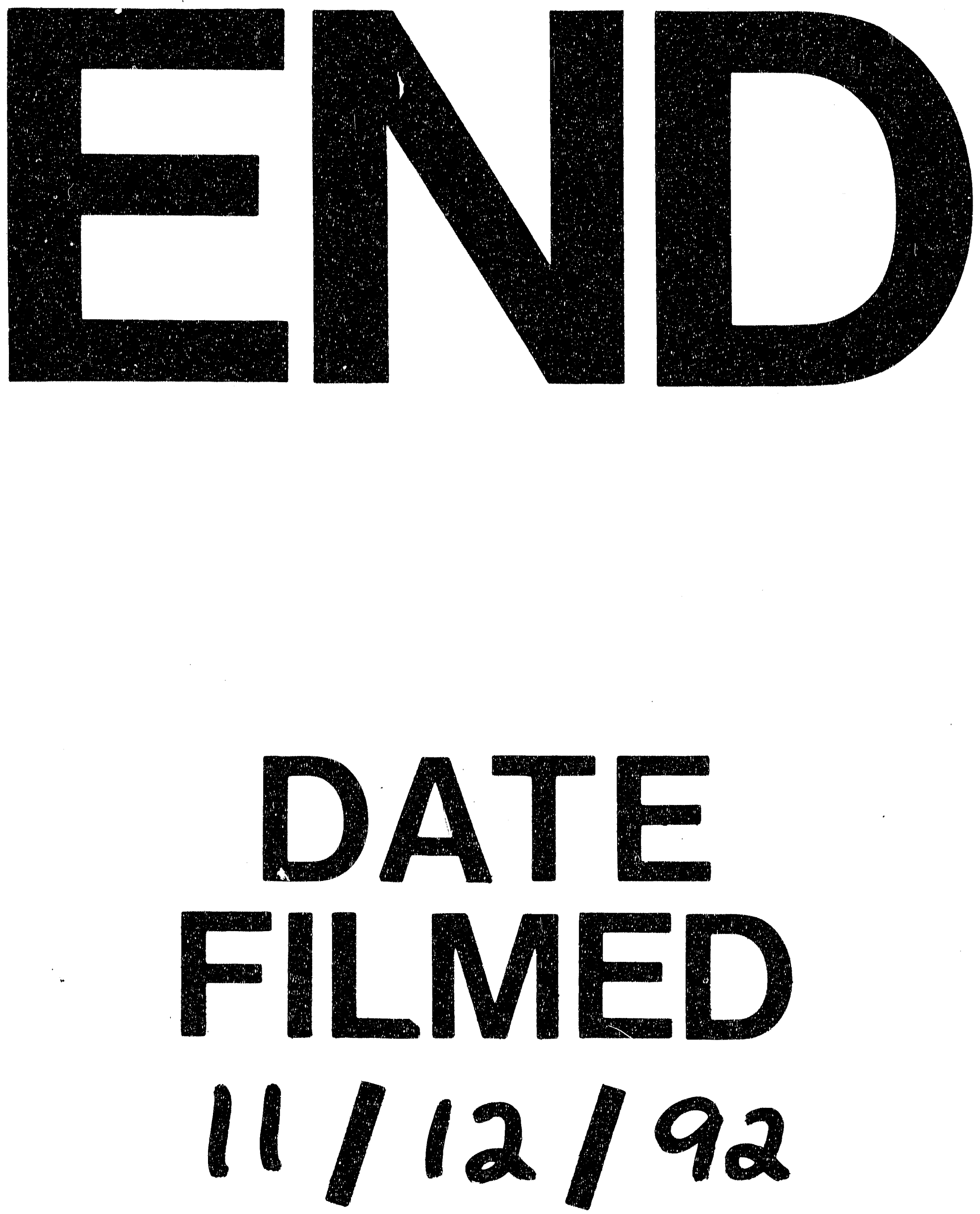

$=$ 
$=$ 